\title{
Associated production of gauge bosons and $D / B$ mesons at the LHCb and double parton interactions
}

\author{
S.P. Baranov, ${ }^{a}$ A.V. Lipatov, ${ }^{b c}$ M.A. Malyshev, ${ }^{b}$ A.M. Snigirev ${ }^{b}$ and N.P. Zotov ${ }^{b \dagger}$ \\ ${ }^{a}$ P.N. Lebedev Institute of Physics \\ Moscow 119991, Russia \\ ${ }^{b}$ Skobeltsyn Institute of Nuclear Physics, Lomonosov Moscow State University \\ Moscow 119991, Russia \\ c Joint Institute for Nuclear Research \\ Dubna 141980, Moscow region, Russia \\ E-mail: baranovesci. lebedev.ru, lipatovatheory.sinp.msu.ru, \\ malyshev@theory.sinp.msu.ru, snigirev@lav01.sinp.msu.ru
}

\begin{abstract}
We study the production of weak gauge bosons in association with heavy flavored mesons at the LHCb conditions in both single (SPS) and double (DPS) parton scattering mechanisms. We find that the usual DPS factorization formula needs to be corrected for the limited partonic phase space, and that including the relevant corrections reduces discrepancies in the associated $Z D$ production. We conclude finally that double parton scattering dominates the production of same-sign $W^{ \pm} D^{ \pm}$ states, as well as the production of $W^{-}$bosons associated with $B$-mesons. The latter processes can thus be regarded as new useful DPS indicators.
\end{abstract}

XXIV International Workshop on Deep-Inelastic Scattering and Related Subjects

11-15 April, 2016

DESY Hamburg, Germany

\footnotetext{
*Speaker.

${ }^{\dagger}$ Deceased
} 
Recently we have shown [1] that the same-sign $W D^{(*)}$ production in ATLAS kinematics region can be an indicative process, in which DPS contribution can dominate over single parton scattering. Our consideration was only restricted to central region because only CMS and ATLAS collaborations provided the data. However, it is interesting to expand our analysis to the forward kinematics region of $\mathrm{LHCb}$, since one can expect even clearer domination of DPS contributions in that region. Also we would like to include into our consideration the processes of $W B$ and $Z D$ production.

Calculation of DPS contributions usually relies on some assumptions concerning the factorization of DPS distribution functions. First, it is supposed, that the DPS distribution function is factorized to transversal and longitudinal parts. Second, the latter reduces to the diagonal product of two independent single parton distribution functions:

$$
D_{p}^{i j}\left(x_{1}, x_{2} ; Q_{1}^{2}, Q_{2}^{2}\right)=D_{p}^{i}\left(x_{1} ; Q_{1}^{2}\right) D_{p}^{j}\left(x_{2} ; Q_{2}^{2}\right)
$$

(here $x_{1}$ and $x_{2}$ are the longitudinal momentum fractions of the partons $i$ and $j$ entering the hard subprocesses at the probing scales $Q_{1}$ and $Q_{2}$ ). These approximations lead to a simple factorization formula (for details see the reviews [2] and references therein):

$$
\sigma_{\mathrm{DPS}}^{A B}=\sigma_{\mathrm{SPS}}^{A} \sigma_{\mathrm{SPS}}^{B} / \sigma_{\mathrm{eff}},
$$

where $\sigma_{\text {eff }}$ is a normalization constant that encodes all "DPS unknowns" into a single phenomenological parameter. However, this formula is valid only for small longitudinal momenta fractions, where the evident restriction on the total parton momenta $x_{1}+x_{2}<1$ can be neglected. This is not the case for the LHCb conditions, especially with respect to as heavy systems as electroweak bosons.

Setting the boundary condition in the form of theta-function $\Theta\left(1-x_{1}-x_{2}\right)$ would result in a step-like discontinuity at the edge of the phase space. This does not seem physically consistent for the parton densities. In a more accurate approach [3-10],

$$
D_{p}^{i j}\left(x_{1}, x_{2} ; Q_{1}^{2}, Q_{2}^{2}\right)=D_{p}^{i}\left(x_{1} ; Q_{1}^{2}\right) D_{p}^{j}\left(x_{2} ; Q_{2}^{2}\right)\left(1-x_{1}-x_{2}\right)^{n},
$$

the kinematical constraints are smoothly put into play with the correction factor $\left(1-x_{1}-x_{2}\right)^{n}$, where $n>0$ is a parameter to be fixed phenomenologically. The integrand and its derivative remain continuous at the phase space border. One often chooses $n=2$. This choice can be partly justified $[3,5]$ in the framework of perturbative QCD and gives DPS distribution functions which satisfy the momentum sum rules [4] reasonably well. To feel the size of the possible effect we also tried $n=3$. A numerical value of $\sigma_{\text {eff }} \simeq 15 \mathrm{mb}$ was earlier obtained empirically from fits to $p \bar{p}$ and $p p$ data. This will be taken as the default value throughout the paper. As we will see, variations within some reasonable range $\sigma_{\text {eff }} \simeq 15 \pm 5 \mathrm{mb}$ would affect our DPS predictions (with the respective errors presented in the tables), though without changing our basic conclusions.

The parameters of our numerical calculations [11] were the following. We employ the $k_{T^{-}}$ factorization approach (see, for instance, [12]) with KMR $k_{T}$-dependent parton distributions [13] for relatively light states $(c \bar{c}$ or $b \bar{b})$ and conventional collinear factorization with MSTW2008 parton densities [14] for states containing $W$ or $Z$ bosons. We used running strong and electroweak coupling constants normalized to $\alpha_{s}\left(m_{Z}^{2}\right)=0.118 ; \alpha\left(m_{Z}^{2}\right)=1 / 128 ; \sin ^{2} \Theta_{W}=0.2312$; the factorization and renormalization scales were chosen as $\mu_{R}^{2}=\mu_{F}^{2}=m_{T}^{2}(W / Z) \equiv m_{W / Z}^{2}+p_{T}^{2}(W / Z)$ for gauge 


\begin{tabular}{lccccc}
\hline channel & data & SPS & DPS $(n=0)$ & DPS $(n=2)$ & DPS $(n=3)$ \\
$Z^{0} D^{0}$ & 2.50 & 0.6 & $2.4 \pm 0.6$ & $1.15 \pm 0.38$ & $0.95 \pm 0.32$ \\
$Z^{0} D^{+}$ & 0.44 & 0.25 & $0.95 \pm 0.32$ & $0.50 \pm 0.17$ & $0.40 \pm 0.13$ \\
sum & 2.94 & 0.85 & $3.35 \pm 0.92$ & $1.65 \pm 0.55$ & $1.35 \pm 0.45$ \\
\hline
\end{tabular}

Table 1: Comparison of the measured and predicted cross-sections (in pb) for $Z$ bosons produced in association with open charm mesons in the fiducial region $p_{T}\left(\mu^{ \pm}\right)>20 \mathrm{GeV}, 2<\eta\left(\mu^{ \pm}\right)<4.5,2<p_{T}(D)<12$ $\mathrm{GeV}, 2<y(D)<4$. The SPS and DPS contributions are shown separately, with $n$ indicating the power of the correction factor (3).

boson production, and $\mu_{R}^{2}=\mu_{F}^{2}=m^{2}(c / b)$ for heavy quark pair production; the quark masses were set to $m_{c}=1.5 \mathrm{GeV}, m_{b}=4.5 \mathrm{GeV}, m_{t}=175 \mathrm{GeV}$. $c$ - and $b$-quarks were converted into $D^{+}$and $B$ mesons using Peterson fragmentation function [15] with $\varepsilon_{c}=0.06$ and $\varepsilon_{b}=0.006$, respectively, and normalized to $f\left(c \rightarrow D^{+}\right)=0.268, f\left(b \rightarrow B^{-}\right)=0.40$ and $f\left(b \rightarrow \bar{B}^{0}\right)=0.40$. For the indirect contributions we also assumed $100 \%$ branching fraction for $t \rightarrow b W$ and used inclusive branching fractions $\operatorname{Br}\left(\bar{B}^{0} \rightarrow D^{+} X\right)=37 \%, \operatorname{Br}\left(B^{0} \rightarrow D^{+} X\right)=3 \%, B r\left(B^{-} \rightarrow D^{+} X\right)=10 \%, B r\left(B^{+} \rightarrow D^{+} X\right)=2.5 \%$.

Now we turn to our numerical results [11]. First, we present our results on the charmassociated $Z$ production [16]. For the LHCb fiducial phase space we obtain $\sigma_{\text {incl }}\left(D^{+}\right)+\sigma_{\text {incl }}\left(D^{0}\right)=$ $670 \mu \mathrm{b}, B r^{Z \rightarrow l l} \sigma_{\text {incl }}\left(Z^{0}\right)=75 \mathrm{pb}$ in excellent agreement with [17] reporting $B r^{Z \rightarrow l l} \sigma_{\text {incl }}\left(Z^{0}\right)=$ $76 \mathrm{pb}$.

We calculate the $Z c \bar{c}$ production cross section at the quark level and then convert $c$-quarks into $D^{0}$ and $D^{+}$mesons with overall probability normalised to $85 \%$ (with the rest $15 \%$ left for $D_{s}$ and $\left.\Lambda_{c}\right)$. We estimate the yields from the different subprocesses as

$$
\begin{aligned}
\sigma(u \bar{u} \rightarrow Z c \bar{c}) & =5 \mathrm{pb}, \\
\sigma(d \bar{d} \rightarrow Z c \bar{c}) & =2.6 \mathrm{pb}, \\
\sigma(g u \rightarrow Z u c \bar{c}) & =11.4 \mathrm{pb}, \\
\sigma(g d \rightarrow Z d c \bar{c}) & =5.2 \mathrm{pb}, \\
\sigma(g g \rightarrow Z c \bar{c}) & =2.5 \mathrm{pb} .
\end{aligned}
$$

Summing up and multiplying by the quark fragmentation probability and by the $Z \rightarrow \mu^{+} \mu^{-}$branching fraction we arrive at $\sigma^{S P S}\left(Z D^{0}, Z D^{+}\right)=0.85 \mathrm{pb}$. This result is consistent with theoretical calculation presented in [16] under the name of 'MCFM massive'. Adding the DPS contribution in the form (2) gives $\sigma^{S P S+D P S}\left(Z D^{0}, Z D^{+}\right)=4.2 \mathrm{pb}$, that significantly exceeds the data. After applying the correction factor (3) the agreement becomes rather satisfactory (see Table 1).

The next considered process is the associated production of $W$ bosons and $D$ mesons. The production of opposite-sign $W^{ \pm} D^{\mp}$ states is dominated by the quark-gluon scattering at $\mathscr{O}\left(\alpha_{s} \alpha\right)$. Among the variety of processes contributing to both opposite-sign and same-sign $W D$ states, the most important ones are the quark-antiquark annihilation at $\mathscr{O}\left(\alpha_{s}^{2} \alpha\right)$ and quark-gluon scattering at $\mathscr{O}\left(\alpha_{s}^{3} \alpha\right)$ (all the processes are listed in Table 2). In addition to that, there present indirect contributions from the production of top-quark pairs $g+g \rightarrow t+\bar{t}$ and $q+\bar{q} \rightarrow t+\bar{t}$ followed by a long chain of decays: $t \rightarrow W^{+} b, W^{+} \rightarrow c \bar{s}, b \rightarrow c X$ or $b \rightarrow c \bar{c} s$ (and the charge conjugated modes). All other possible processes are suppressed by extra powers of coupling constants or by 


\begin{tabular}{lcccc}
\hline \multicolumn{5}{c}{ Double parton scattering contributions } \\
subprocess & $W^{+} D^{+}$ & $W^{+} D^{-}$ & $W^{-} D^{-}$ & $W^{-} D^{+}$ \\
$g g \rightarrow c \bar{c}, \quad u \bar{d} \rightarrow W^{+}$ & $12.3 \pm 4.1$ & $12.3 \pm 4.1$ & - & - \\
$g g \rightarrow c \bar{c}, d \bar{u} \rightarrow W^{-}$ & - & - & $8.9 \pm 3.0$ & $8.9 \pm 3.0$ \\
\hline \multicolumn{5}{c}{ Single parton scattering contributions } \\
subprocess & $W^{+} D^{+}$ & $W^{+} D^{-}$ & $W^{-} D^{-}$ & $W^{-} D^{+}$ \\
$g \bar{s}, g \bar{d} \rightarrow W \bar{c}$ & - & 1.7 & - & - \\
$g s, g d \rightarrow W c$ & - & - & - & 2.0 \\
$u \bar{d} \rightarrow W c \bar{c}$ & 0.8 & 0.8 & - & - \\
$d \bar{u} \rightarrow W c \bar{c}$ & - & - & 0.4 & 0.4 \\
$g u \rightarrow W d c \bar{c}$ & 1.9 & 1.9 & - & - \\
$g \bar{d} \rightarrow W \bar{u} c \bar{c}$ & 0.16 & 0.16 & - & - \\
$g d \rightarrow W u c \bar{c}$ & - & - & 0.8 & 0.8 \\
$g \bar{u} \rightarrow W \bar{d} c \bar{c}$ & - & - & 0.14 & 0.14 \\
$g g \rightarrow t \bar{t} \rightarrow$ decays & 0.01 & 0.01 & 0.01 & 0.01 \\
$q \bar{q} \rightarrow t \bar{t} \rightarrow$ decays & 0.015 & 0.02 & 0.015 & 0.02 \\
\hline
\end{tabular}

Table 2: Predicted $W D$ production cross sections times the $W \rightarrow l v$ branching (in pb) integrated over the fiducial region $p_{T}(l)>20 \mathrm{GeV}, 2<\eta(l)<4.5,2<p_{T}(D)<12 \mathrm{GeV}, 2<\eta(D)<4$.

Kobayashi-Maskawa mixing matrix. Subprocesses $q \bar{q} \rightarrow W^{-} c \bar{s}$ and $q \bar{q} \rightarrow W^{+} s \bar{c}$, though formally of the same order as $q \bar{q} \rightarrow W c \bar{c}$, are heavily suppressed by the gluon propagator having vitrtuality of order $m_{W}^{2}$ rather than $m_{c c}^{2}$.

The individual inclusive SPS cross sections $\sigma\left(D^{ \pm}\right)$and $\sigma\left(W^{ \pm}\right)$for the LHCb fiducial phase space are $\sigma_{\text {incl }}\left(D^{ \pm}\right)=190 \mu \mathrm{b}, B r^{W \rightarrow l v} \sigma_{\text {incl }}\left(W^{+}\right)=970 \mathrm{pb}$ and $B r^{W \rightarrow l v} \sigma_{\text {incl }}\left(W^{-}\right)=680 \mathrm{pb}$, which is in good agreement with [16] and [18], respectively. Our results for SPS and DPS channels are displayed in Table 2. All DPS contributions are presented there without phase space corrections; they have to be multiplied by a correction factor of 0.48 for $n=2$ or 0.38 for $n=3$.

The indirect contributions can be suppressed in experimental analyses using the property that the secondary $b$-decay vertex is displaced with respect to the primary interaction vertex. Summing the direct contributions up, we see that the predicted same-sign $W D$ production rates with and without DPS channels differ by a significant factor. So the forthcoming LHCb data can give conclusive evidence for DPS.

Finally, we present our results on $W B$ associated production. In contrast with the $W D$ production, the contribution, analogous to the process $q g \rightarrow W c$, is absent, and the feed-down from top-quark decays now should be regarded as direct contribution. The full list of processes included in the present analysis is presented in Table 3.

With the parameter setting described above, we estimate the inclusive production of $b$ quarks in the LHCb domain as $\sigma_{\text {incl }}(b)=\sigma_{\text {incl }}(\bar{b})=95 \mu \mathrm{b}$. Combining this result with results for $W$ production we obtain the DPS cross section for $W b$. Table 3 represents our predictions for unconstrained phase space of Eq. (2); they have to be corrected by a factor of 0.45 for $n=2$ or 0.36 for $n=3$. 


\begin{tabular}{lcccc}
\hline \multicolumn{5}{c}{ Double parton scattering contributions } \\
subprocess & $W^{+} B^{+}$ & $W^{+} B^{-}$ & $W^{-} B^{-}$ & $W^{-} B^{+}$ \\
$g g \rightarrow b \bar{b}, \quad u \bar{d} \rightarrow W^{+}$ & $5.5 \pm 1.8$ & $5.5 \pm 1.8$ & - & - \\
$g g \rightarrow b \bar{b}, d \bar{u} \rightarrow W^{-}$ & - & - & $4.0 \pm 1.3$ & $4.0 \pm 1.3$ \\
\hline \multicolumn{5}{c}{ Single parton scattering contributions } \\
subprocess & $W^{+} B^{+}$ & $W^{+} B^{-}$ & $W^{-} B^{-}$ & $W^{-} B^{+}$ \\
$u \bar{d} \rightarrow W b \bar{b}$ & 1.2 & 1.2 & - & - \\
$d \bar{u} \rightarrow W b \bar{b}$ & - & - & 0.5 & 0.5 \\
$g u \rightarrow W d b \bar{b}$ & 2.7 & 2.7 & - & - \\
$g \bar{d} \rightarrow W \bar{u} b \bar{b}$ & 0.22 & 0.22 & - & - \\
$g d \rightarrow W u b \bar{b}$ & - & - & 1.1 & 1.1 \\
$g \bar{u} \rightarrow W \bar{d} b \bar{b}$ & - & - & 0.2 & 0.2 \\
$g g \rightarrow t \bar{t} \rightarrow W W b \bar{b}$ & 0.030 & 0.045 & 0.030 & 0.045 \\
$q \bar{q} \rightarrow t \bar{t} \rightarrow W W b \bar{b}$ & 0.055 & 0.060 & 0.055 & 0.060 \\
$u \bar{d} \rightarrow t \bar{b} \rightarrow W b \bar{b}$ & 0.0018 & 0.0042 & 0.0018 & 0.0042 \\
$d \bar{u} \rightarrow b \bar{t} \rightarrow W \bar{b} b$ & 0.0002 & 0.0005 & 0.0002 & 0.0005 \\
\hline
\end{tabular}

Table 3: Predicted $W B$ production cross sections times the $W \rightarrow l v$ branching (in pb) integrated over the fiducial region $p_{T}(l)>20 \mathrm{GeV}, 2<\eta(l)<4.5,2<\eta(B)<4.5$. Here $B^{+}$and $B^{-}$denote the sum of $B^{+}$ and $B^{0}$ and the sum of $B^{-}$and $\bar{B}^{0}$ mesons, respectively.

In conclusion, the correction factor $\left(1-x_{1}-x_{2}\right)^{n}$, allowing to take into account effects of the limited partonic phase space, gives significant suppression (to a factor of 2) of the $W / Z+D / B$ DPS production cross section; the production of same-sign $W^{ \pm} D^{ \pm}$states in the forward region is dominated by the DPS mechanism, so this process can be recommended as a DPS indicator; LHCb kinematics opens doors for a still new indicative process, which is the beauty-associated production of gauge bosons $W$.

\section{Acknowledgments}

The authors would like to thank I. Belyaev for useful discussions. M.A.M. is grateful to the organizing committee for support. This work was supported in part by RFBR grant 16-32-00176mol-a, grant of the President of Russian Federation NS-7989.2016.2, and by the DESY Directorate in the framework of Moscow-DESY project on Monte-Carlo implementations for HERA-LHC.

\section{References}

[1] S. P. Baranov, M. A. Malyshev, A. V. Lipatov, A. M. Snigirev, and N. P. Zotov, Associated $W^{ \pm} D^{(*)}$ production at the LHC and prospects to observe double parton interactions, Phys. Lett. B 746 (2015) $100[1503.06080[\mathrm{hep}-\mathrm{ph}]]$.

[2] P. Bartalini et al., Multi-Parton Interactions at the LHC, arXiv:1111.0469[hep-ph]; H. Abramowicz et al., Summary of the Workshop on Multi-Parton Interactions (MPI@LHC 2012), arXiv:1306.5413[hep-ph]; S. Bansal et al., Progress in Double Parton Scattering Studies, arXiv:1410.6664[hep-ph]. 
[3] V. L. Korotkikh and A. M. Snigirev, Double parton correlations versus factorized distributions, Phys. Lett. B 594 (2004) 171 [hep-ph/ 0404155 ].

[4] J. R. Gaunt and W. J. Stirling, Double Parton Distributions Incorporating Perturbative QCD Evolution and Momentum and Quark Number Sum Rules, JHEP 1003 (2010) 005 [0910.4347[hep-ph]].

[5] A. M. Snigirev, Asymptotic behavior of double parton distribution functions, Phys. Rev. D 83 (2011) 034028 [1010.4874[hep-ph]].

[6] H.-M. Chang, A. V. Manohar, and W. J. Waalewijn, Double Parton Correlations in the Bag Model, Phys. Rev. D 87 (2013) 034009 [1211.3132 [hep-ph]].

[7] M. Rinaldi, S. Scopetta, and V. Vento, Double parton correlations in constituent quark models, Phys. Rev. D 87 (2013) 114021 [1302.6462 [hep-ph]].

[8] K. Golec-Biernat and E. Lewandowska, How to impose initial conditions for QCD evolution of double parton distributions? Phys. Rev. D 90 (2014) 014032 [1402.4079 [hep-ph]].

[9] F. A. Ceccopieri, A second update on double parton distributions, Phys. Lett. B 734 (2014) 79 [1403.2167[hep-ph]].

[10] A. M. Snigirev, N. A. Snigireva, and G. M. Zinovjev, Perturbative and nonperturbative correlations in double parton distributions, Phys. Rev. D 90 (2014) 014015 [1403.6947 [hep-ph] ] .

[11] S. P. Baranov, M. A. Malyshev, A. V. Lipatov, A. M. Snigirev, and N. P. Zotov, Associated production of electroweak bosons and heavy mesons at LHCb and prospects to observe double parton interactions, Phys. Rev. D 93 (2016) 094013 [1604.03025 [hep-ph]].

[12] B. Andersson et al. (Small x Collab.), Small x phenomenology: Summary and status, Eur. Phys. J. C 25 (2002) 77 [hep-ph/ 0204115 ] ; J. Andersen et al. (Small x Collab.), Small x phenomenology: Summary and status, Eur. Phys. J. C 35 (2004) 67 [hep-ph/0312333]; Small x Phenomenology: Summary of the 3rd Lund Small $x$ Workshop in 2004, Eur. Phys. J. C 48 (2006) 53 [hep-ph/0604189].

[13] M. A. Kimber, A. D. Martin, and M. G. Ryskin, Unintegrated parton distributions, Phys. Rev. D 63 (2001) 114027 [hep-ph/0101348].

[14] A. D. Martin, W. J. Stirling, R. S. Thorne, and G. Watt, Parton distributions for the LHC, Eur. Phys. J. C 63 (2009) 189 [0901.0002[hep-ph]].

[15] C. Peterson, D. Schlatter, I. Schmitt, and P. M. Zerwas, Scaling Violations in Inclusive $e^{+} e^{-}$ Annihilation Spectra, Phys. Rev. D 27 (1983) 105.

[16] R. Aaij et al. (LHCb Collab.), Observation of associated production of a $Z$ boson with a $D$ meson in the forward region, JHEP 1404 (2014) 091 [1401.3245[hep-ex]].

[17] R. Aaij et al. (LHCb Collab.), Measurement of the cross-section for $Z \rightarrow e^{+} e^{-}$production in $p p$ collisions at $\sqrt{s}=7 \mathrm{TeV}$, JHEP 1302 (2013) 106 [1212.4620[hep-ex]].

[18] R. Aaij et al. (LHCb Collab.), Inclusive $W$ and $Z$ production in the forward region at $\sqrt{s}=7 \mathrm{TeV}$, JHEP 1206 (2012) 058 [1204.1620 [hep-ex] ]; Measurement of the forward $W$ boson cross-section in pp collisions at $\sqrt{s}=7 \mathrm{TeV}$, JHEP 1412 (2014) 079 [1408.4354 [hep-ex] ] . 